\title{
Reconsidering Discharge Criteria in Children With Neurologic Impairment and Acute Respiratory Infections
}

\author{
Marie E Wang, MD, MPH, JoAnna K Leyenaar, MD, PhD, MPH², Luci Leykum, MD³
}

\begin{abstract}
${ }^{1}$ Division of Pediatric Hospital Medicine, Stanford University School of Medicine, and Lucile Packard Children's Hospital Stanford, Stanford, California; ${ }^{2}$ Department of Pediatrics and the Dartmouth Institute for Health Policy \& Clinical Practice, Dartmouth-Hitchcock Medical Center, Lebanon, New Hampshire; ${ }^{3}$ Department of Medicine, Dell Medical School, the University of Texas at Austin, and South Texas Veterans Health Care System, San Antonio, Texas.
\end{abstract}

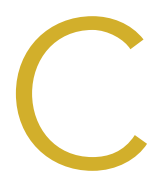

hildren with medical complexity account for $30 \%$ of pediatric hospitalizations and half of all pediatric hospital costs. 'They frequently experience long lengths of stay (LOS), which are associated with hospital-acquired infections, high costs, and family stress.

In this issue of the Journal of Hospital Medicine, Steuart and colleagues investigate one opportunity to decrease LOS in a subset of children with medical complexity by studying the impact of discharge before patients' return to their respiratory baseline status. ${ }^{2}$ They examined 632 hospitalizations in children with neurologic impairment who required increased respiratory support for acute respiratory infections. After adjustment for demographic characteristics, clinical complexity, and acute illness severity, there was no difference in the risk of 30-day hospital reutilization (ie, emergency department revisits and readmissions) when comparing the $30 \%$ of children discharged before returning to their respiratory baseline with the $70 \%$ discharged at baseline (reutilization rates of $32.8 \%$ and $31.8 \%$, respectively).

Twenty-six percent required readmission. This rate is four times that reported for children overall, and higher than the rate for children with the top 10 chronic conditions (range, 6\%$21 \%){ }^{3}$ It also exceeds the median 30 -day risk-standardized readmission rates for adult conditions targeted by the Centers for Medicare \& Medicaid Services (range, 12\%-22\%). ${ }^{4}$ The high readmission rate demonstrates the vulnerability of this population and their need for support in hospital-to-home transitions.

These results suggest important areas for future research. First, the findings need to be replicated by multicenter studies to better understand their generalizability. Second, we need more information about the respiratory support required at discharge, which was not captured in this study. For example, clinicians and families may be more comfortable with discharge for a patient who needs slightly higher levels of their baseline support rather than a new modality of respiratory support. Third, we need to better understand the home context of patients discharged before return to respiratory baseline. Lack of home nursing, in particular, has been associated with discharge delays and prolonged LOS in this population.

*Corresponding Author: Marie Wang, MD, MPH; Email: marie.wang@stanford. edu; Telephone: 650-736-4423; Twitter: @MarieWangMD.

Received: June 28, 2020; Revised: June 29, 2020; Accepted: June 30, 2020

๑) 2020 Society of Hospital Medicine DOI 10.12788/jhm.3496
This study prompts reconsideration of discharge criteria for acute respiratory infections, which often include return to respiratory baseline. Discharge before respiratory baseline for healthy children with bronchiolitis who were discharged on home supplemental oxygen has been associated with shorter hospitalizations and lower costs without differences in reutilization. ${ }^{5}$ Steuart and colleagues demonstrate the potential of this approach in children with neurologic impairment. One key question remains: Which children are most appropriate for discharge before return to respiratory baseline? Family engagement in discussions of goals of hospitalization, self-efficacy, and discharge readiness are important. ${ }^{6}$ These conversations provide context that informs discharge decisions. If the patient is stable and both the medical team and family are comfortable with discharge before respiratory baseline, there may be opportunities to engage in shared decision-making around discharge criteria.

The vulnerability of this population, evidenced by their high rates of readmission, reinforces the importance of family engagement, understanding these children's diverse needs, and further research to identify effective interventions to support safe transitions from hospital to home.

Disclosures: Dr Leyenaar provides consultative services to the American Board of Pediatrics Foundation, not associated with this manuscript. Drs Wang and Leykum have no disclosures.

\section{References}

1. Gold JM, Hall M, Shah SS, et al. Long length of hospital stay in children with medical complexity. J Hosp Med. 2016;11(11):750-756. https://doi. org/10.1002/jhm.2633

2. Steuart $R$, Tan R, Melink K, et al. Discharge before return to respiratory baseline in children with neurologic impairment. J Hosp Med. 2020; 15:531-537. https://doi.org/10.12788/jhm.3394

3. Berry JG, Toomey SL, Zaslavsky AM, et al. Pediatric readmission prevalence and variability across hospitals. JAMA. 2013;309(4):372-380. https://doi. org/10.1001/jama.2012.188351

4. 2017 Medicare Hospital Quality Chartbook. Centers for Medicare \& Medicaid Services. Last updated February 11, 2020. Accessed June 18, 2020. https: //www.cms.gov/Medicare/Quality-Initiatives-Patient-Assessment -Instruments/HospitalQualitylnits/OutcomeMeasures

5. Sandweiss DR, Mundorff MB, Hill T, et al. Decreasing hospital length of stay for bronchiolitis by using an observation unit and home oxygen therapy. JAMA Pediatr. 2013;167(5):422-428. https://doi.org/10.1001/jamapediatrics. 2013.1435

6. Leyenaar JK, O'Brien ER, Leslie LK, Lindenauer PK, Mangione-Smith RM. Families' priorities regarding hospital-to-home transitions for children with medical complexity. Pediatrics. 2017;139(1):e20161581. https://doi. org/10.1542/peds.2016-1581 